\title{
Direct Force Reflecting Teleoperation with a Flexible Joint Robot
}

\author{
Andreas Tobergte and Alin Albu-Schäffer
}

\begin{abstract}
This paper presents a high fidelity force feedback teleoperation control for surgical applications. Advanced control methods, such as flexible joint tracking control and passivity observation, are introduced in the direct force reflecting control architecture. A full state feedback controller of the flexible joint slave robot controls the motor position, velocity, the joint torque, and the torque derivative. The pose of the haptic device and the first three derivatives are observed to generate reference states for the robot control using the robot's inverse dynamics model. Interaction forces of the slave and the environment are measured with a force/torque sensor and directly sent back to the master device. Stability is guaranteed with a passivity observer that monitors the energy in the teleoperation system online and disconnects master and slave if the system operates beyond its stable region. The proposed control architecture is implemented with the sigma.7 haptic device and the MIRO robot. It is experimentally shown, that appropriately considering elasticities with full state reference and control of the slave, increases the dynamic range of the system enabling transparent and stable interaction with hard and soft environments.
\end{abstract}

\section{INTRODUCTION}

Minimally invasive surgery is an emerging field for teleoperation systems, where the body surface constitutes a barrier. In manual minimally invasive surgery the entry point into the human body leads to the loss of two Degree of Freedom (DoF) and hapic perception is disturbed. Furthermore, the surgeon is forced to stand in an unergonomic posture. A telerobotic system that aims to overcome these drawbacks is the MiroSurge system from the German Aerospace Center (DLR) [4], [5], where the surgeon can operate the robotic instruments inside the patient in an intuitive manner from an operator station, as shown in Fig. 1. Mechatronic components of MiroSurge are the light weight robot MIRO that integrates torque sensing in the flexible joints [23], the MICA instruments with force/torque sensing in the tip [21], [17], and the sigma.7 haptic device [22]. The sigma.7 was designed by Force Dimension meeting DLR specifications. It is fully actuated in six DoF and the grasping unit. The DLR version also integrates a force/torque sensor.

Control design for such a robotic system with haptic feedback is challenging. Most popular bilateral control structures of practical interest are the position error based (PEB) control and the direct force reflecting (DFR) control. In PEB control the force feedback is based on the position error between master and slave, which can be interpreted as a virtual spring (and damper). An advantage of this structure is that it is inherently passive because the whole system can be modeled as

\footnotetext{
Andreas Tobergte and Alin Albu-Schäffer, Institute of Robotics and Mechatronics, DLR - German Aerospace Center, Wessling, Germany andreas.tobergteddlr.de, alin.albu-schaefferedlr.de
}

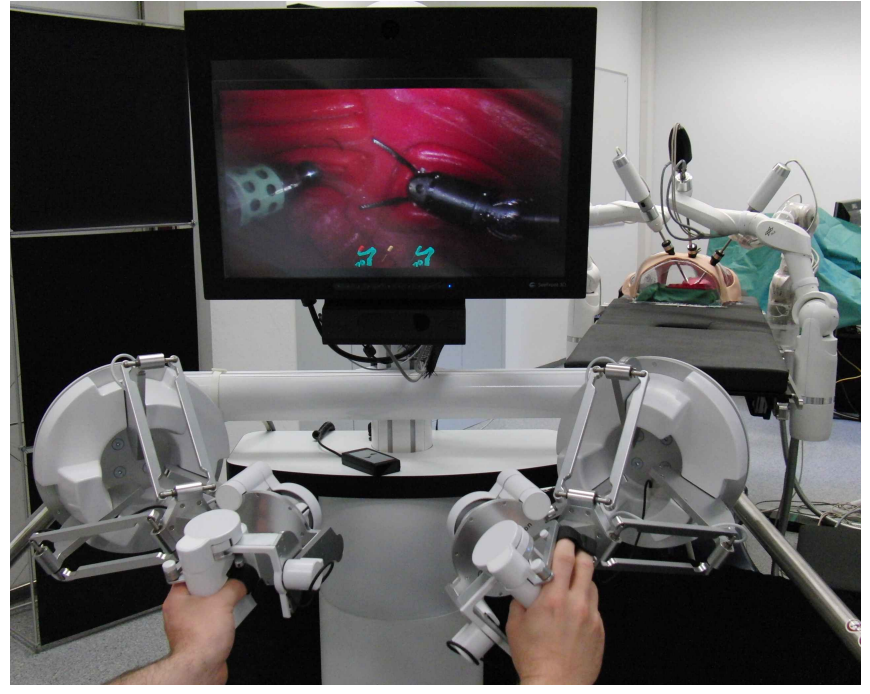

Fig. 1. Foreground: Operator console with two sigma.7 haptic devices and an auto-stereoscopic display; Background: Three MIRO arms with flexible joints attached to an operating table, two of them holding MICA instruments and one holding a stereo endoscope

an interconnection of passive sub-systems [19]. However, the drawback of this approach is that the human operator feels the dynamics of the slave robot. This is a drawback especially if the dynamics of the slave robot is large compared to the environment forces, as it is the case in surgical robotics with soft tissue interaction. Therefore, the DFR approach with a force sensor in the surgical instrument is usually favored for medical applications [12], [11]. In DFR control position (and velocity) commands are sent from the master to a local controller of the slave and measured interaction forces are sent back to the master. It hides the dynamics of the slave robot and the human operator can feel the environment more directly, increasing transparency. The drawback is that the passivity properties of the PEB approach are lost because the velocities and forces exchanged by the master and the slave are not collocated. As a consequence, DFR control shows stability problems in hard contacts [9], [6], [3] Stability is depending on controller gains and environment properties [18], [24]. Studies in literature are usually limited to the one DoF case and mostly refer to rigid robots. Flexible joint models of the slave robot are considered in very few publications, e.g. in [20], [2].

The control approach proposed in this paper aims to combine performance and passivity. Full state reference and full state control of a flexible joint robot is introduced in the DFR bilateral control architecture. The hypothesis of the 
design is that the lower the error between master and slave velocity, the lower the energy that is generated by the teleoperation system. Assuming ideal tracking, where the slave's link velocity always matches the master's velocity, let's the force at the slave's tip become collocated with the master's velocity. The slave virtually vanishes and the DFR control becomes passive like the PEB control. However, perfect tracking is impossible in practice. The control design aims to allow for transparent and stable interaction with a large range of environments by keeping the tracking error low. Since, stability can not be guaranteed for all environments a passivity observer is used to monitor the energy going in and coming out of the teleoperation system. Passivity observers were introduced in haptics and teleoperation primarily in combination with an adaptive damping element, the passivity controller, to dissipate energy generated by the time delay of a communication channel [7] [14]. In the approach presented here, the passivity observer activates a clutch, disconnecting master and slave, if the teleoperation system generates energy. However, the controller is designed to be stable for the expected tasks and environments.

In section II the observation of the master's pose up to the third derivative is described. Section III depicts the generation of reference states for the robot controller. These methods are integrated into a bilateral control scheme in section IV. The control approach is experimentally validated in section V. Section VI concludes the paper.

\section{HAPTIC DEVICE MOTION OBSERVER}

The sigma.7 haptic device comprises three main components: translational base (3 DoF), rotational wrist extension (3 DoF), and grasping unit (1 DoF). It is fully actuated in seven DoF and integrates a force/torque sensor in between the last axis of the wrist and the grasping unit. Translations and rotations of the device are kinematically and statically completely decoupled. The dynamics is strongly decoupled with the inertia matrix being nearly diagonal [22].

The dynamics of the sigma.7 is split into two parts separated by the force/torque sensor. The first part is the translational base and the rotational wrist responsible for the 6 Dof motion and actuation in space. The second part is the handle with the grasping, which is a separated functional DoF. The handle is consequently considered to be a passive mass attached to the force/torque sensor.

The dynamic model for the translational base and the rotational wrist is given with,

$$
\left(\mathbf{B}+\mathbf{M}_{l}(\boldsymbol{\theta})\right) \ddot{\theta}+\mathbf{C}_{l}(\boldsymbol{\theta}, \dot{\theta}) \dot{\theta}+\mathbf{g}_{l}(\boldsymbol{\theta})+\tau_{s}=\tau_{m}
$$

where $\theta \in \mathfrak{R}^{6}$ represents the motor joint angles. The motor torque $\tau_{m}$ is actuating the motor inertia $\mathbf{B}$ that is assumed to be rigidly connected with the link inertia $\mathbf{M}_{l}$. The model is completed with the centripetal-/coriolis torques $\mathbf{C}_{l}(\theta, \dot{\theta}) \dot{\theta}$ and gravity $\mathbf{g}_{l}$. Friction is not considered here.

The sensor torque in joint space

$$
\tau_{s}=\mathbf{J}(\theta)^{T} \mathbf{w}_{s, M}
$$

is equivalent to the wrench (force and torques in $6 \mathrm{DoF}$ ) of the force/torque sensor $\mathbf{w}_{s, M}$ transformed with the jacobian $\mathbf{J}(\theta){ }^{1}$

\section{A. Observation of acceleration}

The master motion in Cartesian space from pose to jerk is $\left(\mathbf{x}_{M}, \dot{\mathbf{x}}_{M}, \ddot{\mathbf{x}}_{M}, \dddot{\mathbf{x}}_{M}\right)$, where the pose $\mathbf{x}_{M}=f k i n(\theta)$ is given with the forward kinematics and measured joint positions. The velocity can be derived from the Cartesian pose or calculated from derived joint velocity with the Jacobian. However, the second derivative of measured variables is usually to noisy to be used in practice. Therefore, an observer for acceleration

$$
\hat{\mathbf{a}}=\left(\mathbf{B}+\mathbf{M}_{l}(\boldsymbol{\theta})\right)^{-1}\left(\tau_{m}-\mathbf{g}_{l}(\boldsymbol{\theta})-\tau_{s}\right)
$$

is defined, by solving the dynamics (1) for the acceleration $\ddot{\theta}$, neglecting centripetal-/coriolis torques. The observation is based on the motor torques $\tau_{m}$ and the measured torques $\tau_{s}$. Cartesian acceleration

$$
\ddot{\mathbf{x}}_{M}=\dot{\mathbf{J}}(\theta) \dot{\theta}+\mathbf{J}(\theta) \hat{\mathbf{a}}
$$

and jerk

$$
\dddot{\mathbf{x}}_{M}=\ddot{\mathbf{J}}(\theta) \dot{\theta}+2 \dot{\mathbf{J}}(\theta) \hat{\mathbf{a}}+\mathbf{J}(\theta) \dot{\hat{\mathbf{a}}}
$$

can be calculated using the chain rule. However, the first term in (5) can be neglected. Second order derivatives of the Jacobian are almost zero and primarily contain noise because they implicitly include the second derivative of position measurements.

\section{B. Master control}

The commanded motor torques of the haptic device

$$
\tau_{m, d}=\mathbf{J}(\theta)^{T} \mathbf{w}_{m, M}+\mathbf{g}_{l}(\boldsymbol{\theta})+\mathbf{g}_{h}(\boldsymbol{\theta})
$$

include the Cartesian wrench sent to the master $\mathbf{w}_{m, M}$ and gravitational torques of the links and the handle. Gravity compensation of the handle $\mathbf{g}_{h}$ is based on $\theta$, because the stiffness of the force/torque sensor is very high and the deformation caused by the sensor is neglectable [22]. The controller (6) can be extended with a term for work space limits in Cartesian space or joint space implemented as virtual springs and dampers.

\section{ROBOT TRACKING CONTROL}

The MIRO is a versatile robot for surgical interventions in open surgery and minimally invasive surgery. It's kinematics are redundant and similar to the one of a human arm. It has a base joint (1 DoF), and coupled joints for the shoulder (2 DoF), elbow (2 DoF) and wrist (2 DoF). The elastic joints are equipped with torque sensors [23].

The robot dynamics is given in joint space, with the motor side dynamics

$$
\tau_{m}=\mathbf{B} \ddot{\theta}+\tau
$$

\footnotetext{
${ }^{1}$ Variables in section II and section III are only locally defined in general. Cartesian variables with capital letter index $M$ (master) or $S$ (slave) are globally defined in one and the same invariant frame of reference. The Jacobians in in section II and section III are given w.r.t this frame.
} 
and the link side dynamics

$$
\tau+\tau_{\text {ext }}=\mathbf{M}(\mathbf{q}) \ddot{\mathbf{q}}+\mathbf{C}(\mathbf{q}, \dot{\mathbf{q}}) \dot{\mathbf{q}}+\mathbf{g}(\mathbf{q})
$$

The joint torques

$$
\tau=\mathbf{K}(\theta-\mathbf{q})
$$

of the flexible joints are the difference of the motor side position $\theta \in \mathfrak{R}^{7}$ and the link side position $\mathbf{q}$, scaled with the stiffness $\mathbf{K}$. The motor side dynamics are represented with the motor torque $\tau_{m}$, the motor inertia $\mathbf{B}$ and acceleration $\ddot{\theta}$, and the joint torque $\tau$. The equations (7)(8)(9) are written in joint space. Due to the joint coupling in the MIRO robot the motor inertia matrix in joint space and the joint stiffness matrix are not diagonal matrices, but rather matrices with one scalar element (base joint) and three $2 \times 2$ matrices, one for each coupled joint (shoulder, elbow, wrist). The link side rigid body dynamics consist of the link inertia $\mathbf{M}(\mathbf{q})$, coriolis- and centripetal torques $\mathbf{C}(\mathbf{q}, \dot{\mathbf{q}}) \dot{\mathbf{q}}$ and gravitational torques $\mathbf{g}(\mathbf{q})$. Motor friction and joint damping is neglected.

\section{A. State feedback control}

The complete state vector $(\theta, \dot{\theta}, \tau, \dot{\tau})^{T}$ of a flexible joint robot can be controlled with

$$
\tau_{c}=\mathbf{K}_{P}\left(\theta_{d}-\theta\right)+\mathbf{K}_{D}\left(\dot{\theta}_{d}-\dot{\theta}\right)+\mathbf{K}_{T}\left(\tau_{d}-\tau\right)+\mathbf{K}_{S}\left(\dot{\tau}_{d}-\dot{\tau}\right)
$$

where $\mathbf{K}_{P}, \mathbf{K}_{D}, \mathbf{K}_{T}, \mathbf{K}_{S}$ are positive definite gains for every state error. The closed loop system emulates the behavior of a passive system, where the torque feedback can be interpreted as a shaping of kinetic energy and the position feedback as a shaping of potential energy [1]. Global asymptotic stability can be shown based on Lyapunov theory, for the case that the system is autonomous, i.e. the reference states are fixed. Details of a full state feedback MIMO-controller respecting joint coupling and elasticity are given in [10].

\section{B. State reference}

A full state command for free space $\left(\tau_{\text {ext }}=0\right)$ tool tip tracking control requires the robot dynamics to be rewritten as functions of the desired link side position $\mathbf{q}_{d}$ and it's derivatives. Rewriting (9) gives the desired motor position

$$
\theta_{d}=\mathbf{q}_{d}+\mathbf{K}^{-1} \tau_{d}
$$

and deriving leads to the motor velocity

$$
\dot{\theta}_{d}=\dot{\mathbf{q}}_{d}+\mathbf{K}^{-1} \dot{\tau}_{d}
$$

The resulting desired torque from (8) is

$$
\tau_{d}=\mathbf{M}\left(\mathbf{q}_{d}\right) \ddot{\mathbf{q}}_{d}+\mathbf{C}\left(\mathbf{q}_{d}, \dot{\mathbf{q}}_{d}\right) \dot{\mathbf{q}}_{d}+\mathbf{g}\left(\mathbf{q}_{d}\right)
$$

and deriving the equation using the chain rule leads to

$$
\dot{\tau}_{d}=\dot{\mathbf{M}}\left(\mathbf{q}_{d}\right) \ddot{\mathbf{q}}_{d}+\mathbf{M}\left(\mathbf{q}_{d}\right) \dddot{\mathbf{q}}_{d}+\dot{\mathbf{C}}\left(\mathbf{q}_{d}, \dot{\mathbf{q}}_{d}\right) \dot{\mathbf{q}}_{d}+\mathbf{C}\left(\mathbf{q}_{d}, \dot{\mathbf{q}}_{d}\right) \ddot{\mathbf{q}}_{d}+\dot{\mathbf{g}}\left(\mathbf{q}_{d}\right)
$$

With (11),(12),(13) and (14) the desired states $\left(\theta_{d}, \dot{\theta}_{d}, \tau_{d}, \dot{\tau}_{d}\right)^{T}$ are given as a function of the desired link side motion $\left(\mathbf{q}_{d}, \dot{\mathbf{q}}_{d}, \ddot{\mathbf{q}}_{d}, \dddot{\mathbf{q}}_{d}\right)^{T}$.

However, in the case that the robot gets in contact with the environment, i.e. $\tau_{\text {ext }} \neq 0$, gravity compensation based on the desired pose is not adequate, since the difference between desired and actual positions is not sufficiently small in general. Therefore, gravity compensation is done based on position measurements of the robot. A estimation of the link side position is $\overline{\mathbf{q}}$, which is statically equivalent to $\mathbf{q}$ and is computed only with the motor side position $\theta$ [13].

The torque command (13) is modified for the general case with environment interaction

$$
\tau_{d}=\mathbf{M}\left(\mathbf{q}_{d}\right) \ddot{\mathbf{q}}_{d}+\mathbf{C}\left(\mathbf{q}_{d}, \dot{\mathbf{q}}_{d}\right) \dot{\mathbf{q}}_{d}+\mathbf{g}(\overline{\mathbf{q}})
$$

so that the dynamics is based on the reference motion but gravity compensation is based on the actual motor side position of the robot's joints. The reference for the torque derivative (14) is consequently modified

$$
\dot{\tau}_{d}=\dot{\mathbf{M}}\left(\mathbf{q}_{d}\right) \ddot{\mathbf{q}}_{d}+\mathbf{M}\left(\mathbf{q}_{d}\right) \dddot{\mathbf{q}}_{d}+\dot{\mathbf{C}}\left(\mathbf{q}_{d}, \dot{\mathbf{q}}_{d}\right) \dot{\mathbf{q}}_{d}+\mathbf{C}\left(\mathbf{q}_{d}, \dot{\mathbf{q}}_{d}\right) \ddot{\mathbf{q}}_{d}
$$

to only include the derivatives of the dynamic terms.

\section{Slave control}

The tracking controller of the flexible slave robot includes references for all states. The commanded motor torque

$$
\tau_{m, d}=\tau_{d}+\tau_{c}\left(\theta_{d}, \dot{\theta}_{d}, \tau_{d}, \dot{\tau}_{d}, \theta, \dot{\theta}, \tau, \dot{\tau}\right)
$$

follows from (7). It includes of a feedforward term of the desired joint torques and the controller term (10), where the state references are defined by (11), (12), (15) and (16). The feedforward term for the motor inertia from (7) was dropped because it depends on the forth derivative of the position and generates a non-causal feedback in the teleoperation system.

\section{Joint space motion reference}

The reference motion for the slave controller $\left(\mathbf{x}_{S, d}, \dot{\mathbf{x}}_{S, d}, \ddot{\mathbf{x}}_{S, d}, \dddot{\mathbf{x}}_{S, d}\right)$ is given in Cartesian space, with the pose and three derivatives. The link side joint positions

$$
\mathbf{q}_{d}=\operatorname{invkin}\left(\mathbf{x}_{S, d}\right)
$$

can be calculated with an analytic inverse kinematics solution [8]. Joint velocities are derived from the joint positions or calculated with the pseudo-inverse Jacobian:

$$
\dot{\mathbf{q}}_{d}=\mathbf{J}^{\#} \dot{\mathbf{x}}_{S, d}
$$

However, the acceleration needs to be calculated separately, because it is observed with additional sensors (3). Using the chain rule leads to the reference acceleration in joint space

$$
\ddot{\mathbf{q}}_{d}=\dot{\mathbf{J}}^{\#} \dot{\mathbf{x}}_{S, d}+\mathbf{J}^{\#} \ddot{\mathbf{x}}_{S, d}
$$

and the reference jerk

$$
\dddot{\mathbf{q}}_{d}=\ddot{\mathbf{J}}^{\#} \dot{\mathbf{x}}_{S, d}+2 \dot{\mathbf{J}}^{\#} \ddot{\mathbf{x}}_{S, d}+\mathbf{J}^{\#} \dddot{\mathbf{x}}_{S, d}
$$

In analogy to (5) the first term in (21) is neglected, because it contains the second derivative of the Jacobian.

\section{BILATERAL CONTROL}

The haptic device and the flexible joint robot are connected to a direct force reflecting teleoperation system. 


\section{A. Direct force reflecting control}

The haptic device with control and motion observation (section II) and the flexible joint robot with full state control (section III) are integrated into a teleoperation system with direct force reflecting control, as shown in Fig. 2.

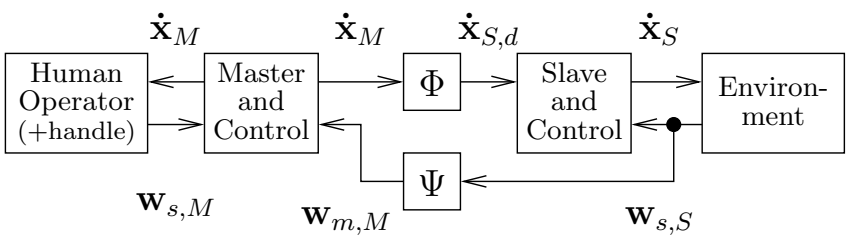

Fig. 2. Direct Force Reflecting Teleoperation with motion and force/torque scaling

Motion commands are sent from the master to the slave. The commanded slave velocity

$$
\dot{\mathbf{x}}_{S, d}=\Phi \dot{\mathbf{x}}_{M} c
$$

equals the master velocity scaled with a positive diagonal matrix $\Phi$. The variable $c \in\{0,1\}$ indicates whether master and slave are connected $(c=1)$ or disconnected $(c=0)$. The commanded acceleration and jerk are calculated the same way as the velocity (22). The reference pose of the slave needs to be initialized with the initial pose of the slave at time $(t=0)$ as starting points such that the the pose reference is smooth and consistend with the velocity. However, initialisation of the system is not described here.

The force/torque command sent to the master

$$
\mathbf{w}_{m, M}=\Psi \mathbf{w}_{S, s} c
$$

is the wrench measured at the slave's tip $\mathbf{w}_{S, s}$ multiplied with with a positive diagonal scaling matrix $\Psi$. In general, if $\Phi$ or $\Psi$ are not identity matrices the teleoperation system is scaled in motion or force, respectively.

\section{B. Passivity Observer}

A major disadvantage of the DFR control as defined above is that no statement about the stability or passivity of the teleoperation system can be made analytically for the general non-linear system interacting with arbitrary environments. Even though the haptic device and the flexible joint robot with their local controllers are passive systems, the bilateral teleoperation system is not passive in general. The velocity sent to the slave $\dot{\mathbf{x}}_{S, d}$ and the wrench at the slave's tip $\mathbf{w}_{S, s}$ sent back to the master are not collocated, i.e. they are not power conjugated and there is no energy defined for these signals. As a consequence the energy exchanged by the master and the slave can not be evaluated using these quantities.

A solution for ensuring passivity of a DFR teleoperation system is proposed based on the time domain passivity approach. The teleoperation system is considered a two port system having one port with the human operator and a second port with the remote environment, as shown in Fig. 3.

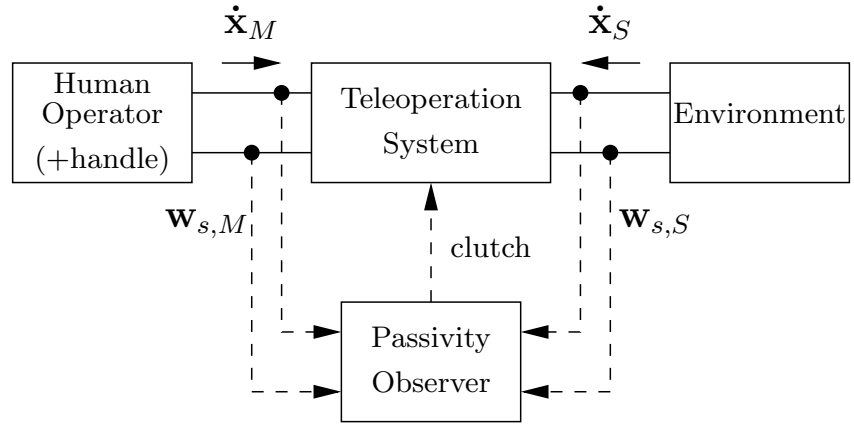

Fig. 3. Passivity observer observing the input/output energy of the teleoperation system

The energy flowing into the two port

$$
E_{\text {in }}(t)=\int_{0}^{t} \mathbf{w}_{s, M}^{T} \dot{\mathbf{x}}_{M}
$$

and the energy flowing out of two port

$$
E_{\text {out }}(t)=\int_{0}^{t}-\mathbf{w}_{S, S}^{T} \dot{\mathbf{x}}_{S}
$$

represent the energy flow of the teleoperation system while interacting with the human operator and the environment. The total energy in the network

$$
E_{N}(t)=E_{\text {in }}(t)+E_{\text {out }}(t)
$$

is the sum of the energy at the ports. The network with initial energy $E_{N}(0)=0$ is passive [7] [16] if and only if

$$
E_{N}(t) \geq 0, \quad \forall t \geq 0
$$

However, the definition of passivity for a two port as the energy balance over an infinite time is troublesome when applied as an indicator for save operation of a mechanical system. The problem lies in the ambiguity of the input and output energy. A mechanical system is not loss less, like e.g. a communication channel. It is not clear whether energy that enters the system is stored as kinetic or potential energy, or dissipated as friction. Complementary, energy leaving the system can be stored energy or generated energy. As a consequence the two port can dissipate an arbitrary amount of energy over a long time and can then generate the same amount of energy before becoming active by definition (27) [15]. Therefore, the energy $E_{N}$ is limited to a maximum level, where the integrator is saturated:

$$
E_{N, \max } \geq E_{N}(t), \quad \forall t \geq 0
$$

The limitation of energy in (28) is complementing the passivity criterion (27) without weakening it, rather making it more conservative. The passivity observer triggers an emergency clutch, $c=0$ in (22),(23), that disconnects master and slave if the teleoperation system becomes active, i.e. the inequality (27) is not fulfilled.

$$
E_{N}(t)<0 \Rightarrow c(t)=0, \quad \forall t \geq 0
$$

Master and slave are then disconnected and are only controlled by their local passivity based controllers. 


\section{EXPERIMENTS}

An experimental system was set up with the sigma.7 haptic device and the flexible joint robot MIRO holding a passive tool with a force/torque sensor attached to the MICA instrument drive unit, as shown in Fig. 4. Experiments were carried out interacting with rigid objects and soft springs. The first experiment evaluates the stability of DFR control with full state reference versus DFR control with position and velocity reference only. The second experiment shows the fidelity of DFR control with full state reference when sliding on a dice. In a third experiment the human operator feels linear springs with different compliances. The experiments were done with translations. The orientation of the wrist of the sigma.7 was held constant in the nominal pose.
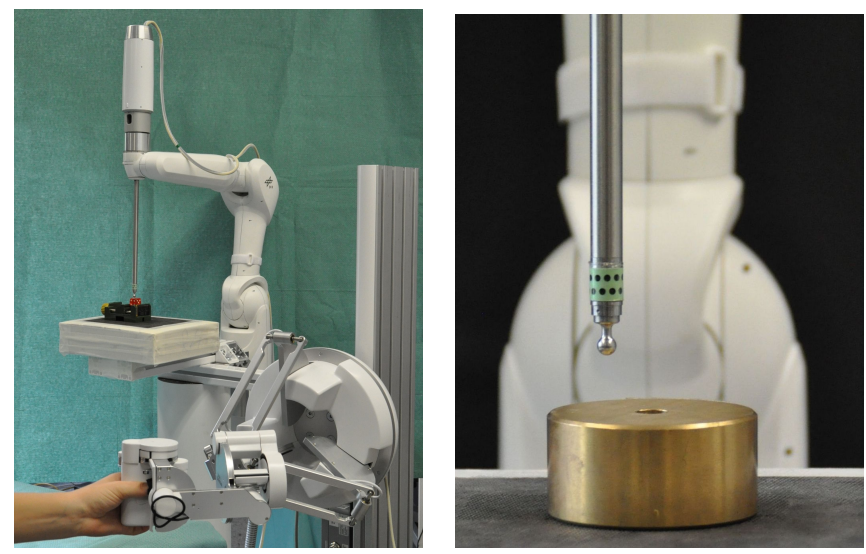

Fig. 4. Left: Test-setup with the sigma.7 in the foreground and the flexible joint robot MIRO holding a passive palpation tool attached to the MICA drive unit; Right: The palpation tool with force/torque sensor interacting with a rigid object

\section{A. Stability in hard contact}

The stability of the controller in hard contacts was evaluated. A small mass of about $m_{h}=0.13 \mathrm{Kg}$ was put on the grasping unit of the sigma.7, causing master and slave to freely fall against a rigid metal block (Fig. 4, right). The stiffness of the metal block and the substrate is $k_{e}>$ $10000 \frac{N}{m}$. The experiment was done with position and velocity command only, i.e. $\ddot{\mathbf{x}}_{S, d}=\dddot{\mathbf{x}}_{S, d}=\mathbf{0}$, and with full state command. It was done in one Cartesian DoF with motion scaling $\Phi=\operatorname{diag}(0,0,0.66,0,0,0)$ and force scaling $\Psi=$ $\operatorname{diag}(0,0,1,0,0,0)$. The results for the DFR control with positon and velocity reference only are shown on the left side in Fig. 5. DFR control with full state command is shown on the right. The z-positions of master (black) and slave (green) are shown in the upper graphs. The master position is scaled with $\Phi$ for easier comparison. It can be seen that the control with full state reference and feedback settles in a stable contact, whereas the teleoperation system without full state reference becomes unstable. The corresponding energy in the teleoperation system $E_{N}$ (26) is shown in the lower graphs. With both control structures $E_{N}$ increases during the free fall, i.e. the teleoperation system stores and/or dissipates energy. However, when getting into contact DFR
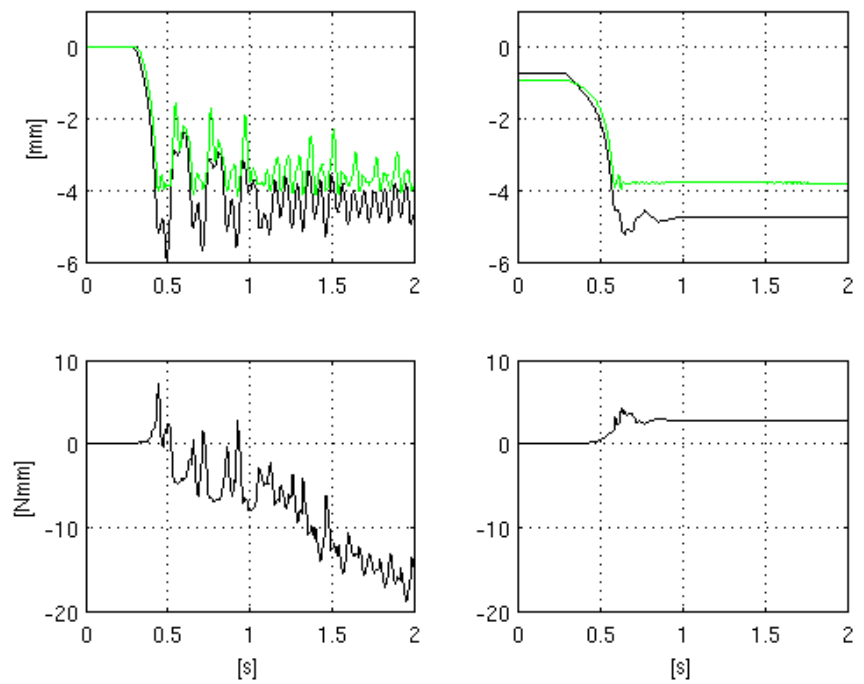

Fig. 5. Free fall with an external load against a hard contact; Left: DFR control with position and velocity command; Right: DFR control with full state command; Top: position of master (black) and slave (green); Bottom: energy in the system

control with full state command releases stored energy and potentially generates a small amount of energy, whereas the control without full state command keeps on generating energy. The energy in the system quickly drops below zero and the instability can be clearly identified by the passivity observer (27). Note, that the passivity clutch was switched off for this experiment.

\section{B. Sliding on rigid structures}

The ability to display rigid surfaces was tested with a dice, as shown in Fig. 6. The human operator was moving the teleoperation system in three Dof with motion scaling $\Phi=\operatorname{diag}(0.25,0.25,0.25,0,0,0)$ and force scaling $\Psi=\operatorname{diag}(2,2,2,0,0,0) .{ }^{2}$ However, the task was rather two dimensional. The human operator was approaching the dice from the left side in Fig. 6 moving upwards on the top surface, sliding to the right, rest in cavity (one is on top of the dice) and keep on sliding to the right down the dice. In the y-z-trajectory of the slave in Fig. 6 can be seen how the tool tip (6 mm diameter) moves into the cavity $(3 \mathrm{~mm}$ diameter) of the dice (17 mm side length).

In Fig. 7 the corresponding positions (top graphs), velocity (middle) and force (bottom) of the y-axis (left) and the z-axis (right) are shown for the part of the trajectory on top of the dice from point $A$ to point $B$. One can clearly see the forward motion in the position of the $y$-axis and the horizontal motion with the cavity of the dice in z-axis position (master black and scaled with $\Phi$; slave green). Even though there is some feedback in the $\mathrm{z}$-axis when entering the cavity $(t \approx 0.9)$ and leaving it $(t \approx 1.8)$ the most sensation is felt in the y-axis by the human operator. This can be seen in the velocity and force of the master $y$-axis. The teleoperation system shows good velocity tracking capabilities in the y-axis, while being

\footnotetext{
${ }^{2}$ This scaling was used for all experiments in the attached video.
} 


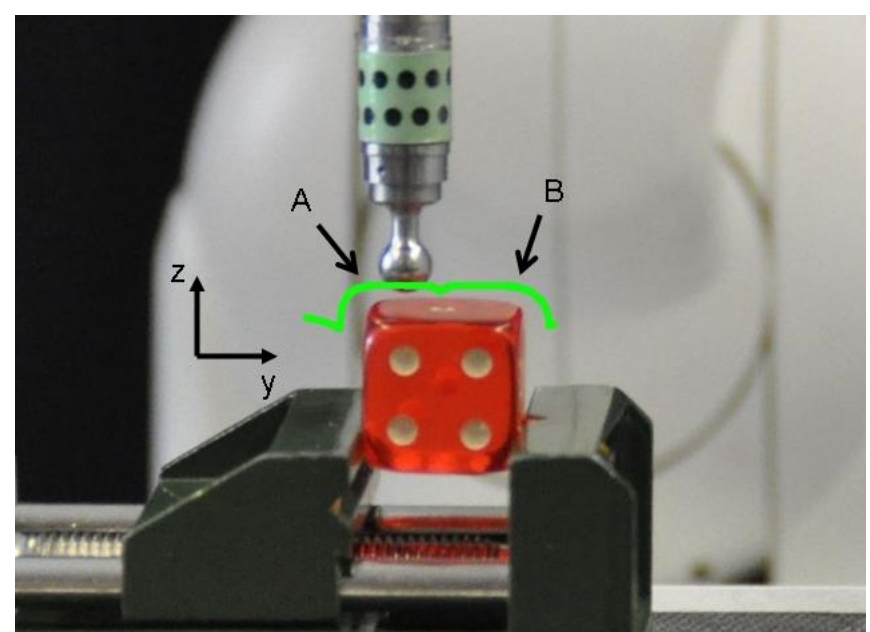

Fig. 6. Trajectory of a human operator palpating a dice with number one on top, sliding from left to right in positive y-axis

TABLE I

STIFFNESS TRANSMISSION

\begin{tabular}{|l||l|l|}
\hline & $\begin{array}{l}\text { environment } \\
\text { stiffness } \\
{[\mathrm{N} / \mathrm{m}]}\end{array}$ & $\begin{array}{l}\text { human } \\
\text { stiffness } \\
{[\mathrm{N} / \mathrm{m}]}\end{array}$ \\
\hline spring 1 & 1540 & 1260 \\
\hline spring 2 & 905 & 786 \\
\hline spring 3 & 687 & 653 \\
\hline spring 4 & 281 & 287 \\
\hline spring 5 & 62 & 64 \\
\hline
\end{tabular}

in a hard contact in the z-axis. The master force in the graph is inverted in the sign. The magnitudes roughly match the measured force at the slave scaled with two.

\section{Stiffness Transmission}

Palpation of tissue is a typical task in surgery, where haptic feedback is beneficial. A human operator was slowly pushing the master device against a linear spring up to a maximum force of about $3 N$ (i.e., $f_{h \text {,steady-state }} \approx 3 N$ ), as shown in Fig. 8. The experiment was done in the $\mathrm{z}$ axis with a 1 -by-1 scaling, i.e. $\Phi=\operatorname{diag}(0,0,1,0,0,0)$ and $\Psi=\operatorname{diag}(0,0,1,0,0,0)$. The slave starts at the contact point, where the positions of master and slave (top graph) are set to zero. The stiffness of the environment (Fig. 8, bottom, green) is estimated

$$
\hat{k}_{e}=\left|\frac{f_{e}}{p_{s}}\right|
$$

with the measured force in the z-axis at the tip $f_{e}$, (Fig. 8, middle) and the position in $\mathrm{z}$-axis at the tip of the slave $p_{s}$, i.e. the link side position. The stiffness displayed to the human operator $\hat{k}_{h}$ is estimated with force and position in zaxis at the master device. The stiffness estimation considers the static case only and shows errors due to dynamic effects when master and slave are moving. When the force at the master reaches the maximum of $3 N$ the force at the slave, the positions and consequently the stiffness estimations settle to constant values.
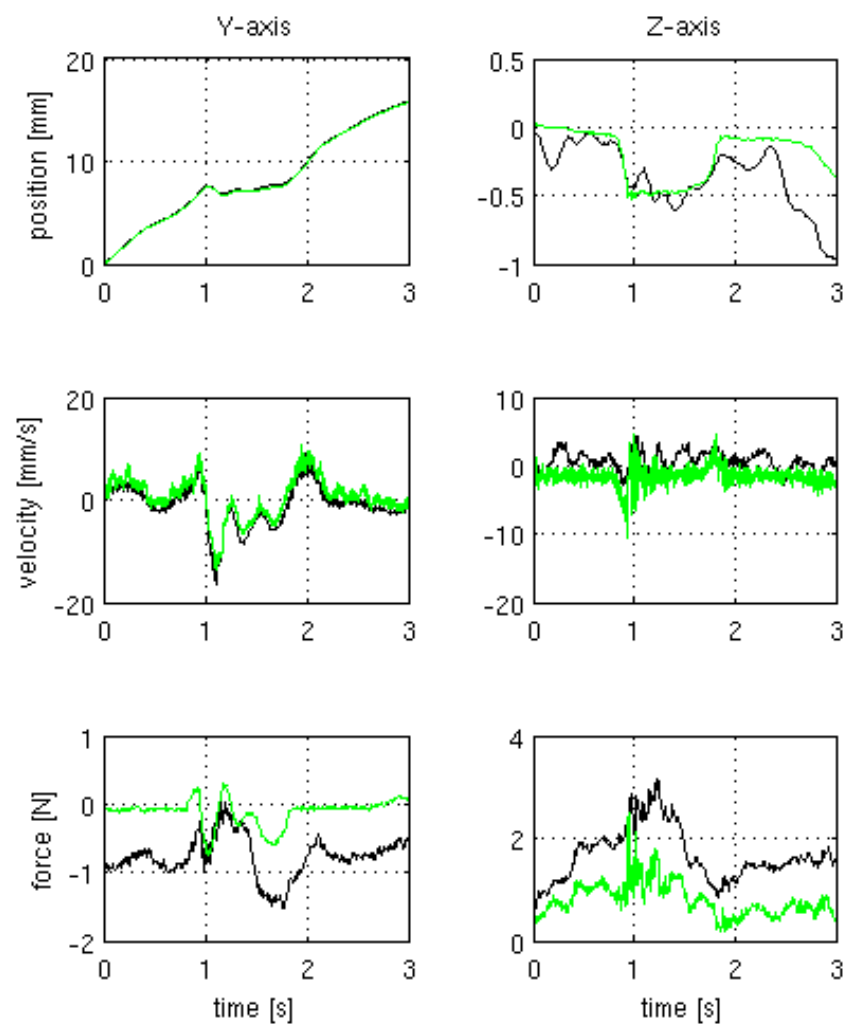

Fig. 7. Human operator touches a dice; Left: y-axis; Right; z-axis; Top positions of master (black) and slave (green); Middle: velocities; Bottom: forces
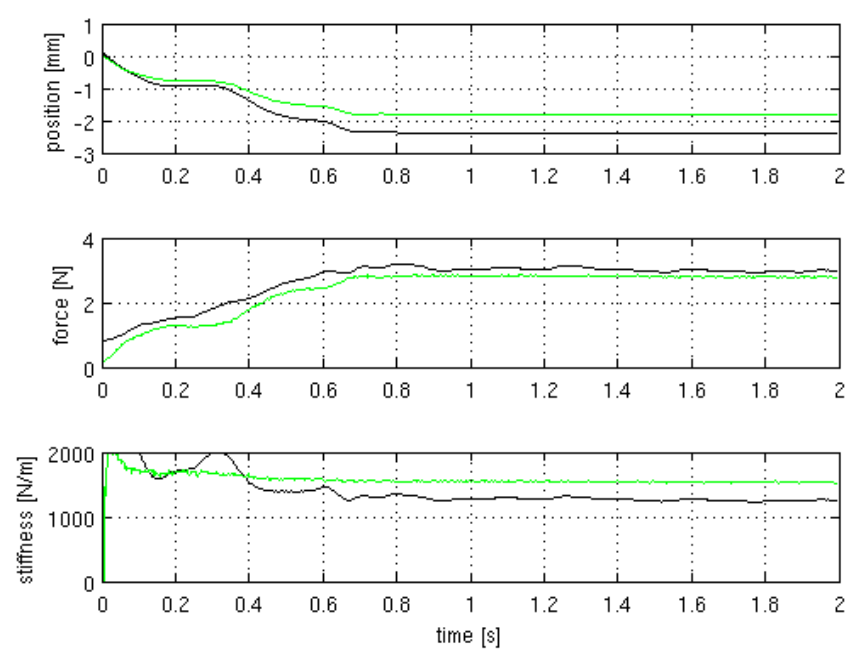

Fig. 8. Stiffness of a spring transmitted to the human operator; Top: positions of master (black) and slave (green); Middle: forces; Bottom: estimated stiffness 
A constant derivation of the environment stiffness $\hat{k}_{e}=$ $1540 \frac{N}{m}$ versus $\hat{k}_{h}=1260 \frac{N}{m}$ of the human operator can be seen. The experiment was repeated with four softer springs, as shown in Table I. The top row with estimations for spring 1 correspond to Fig. 8. Smaller environment stiffnesses are displayed accurately to the human operator, whereas derivations occur for more rigid environments. This is primarily caused by the compliance of the slave robot. The expected stiffness that is transmitted to the human operator

$$
k_{h}=\left(\frac{1}{k_{e}}+\frac{1}{k}+\frac{1+k_{t}}{k_{p}}\right)^{-1}
$$

is given with the steady-state model of the teleoperation system for the z-axis linearized at the contact point. The model contains the joint stiffness $k=20700 \frac{N}{m}$ and the controller stiffness with the proportional position gain $k_{p}=46600 \frac{N}{m}$ and the torque feedback gain $k_{t}=5.26 \frac{\mathrm{N}}{N}$.

In Fig. 9 the stiffness transmission from the environment (abscissa) to the human operator (ordinate) is shown. The expected stiffness transmitted $k_{h}$ (solid line) is close to the ideal 1-by-1 transmission (dashed line) and nearly linear for stiffnesses below $1000 \frac{\mathrm{N}}{\mathrm{m}}$, which is the most relevant range for soft tissue interaction. The diamonds show the experimental results for spring 1 to 5 of Table I.

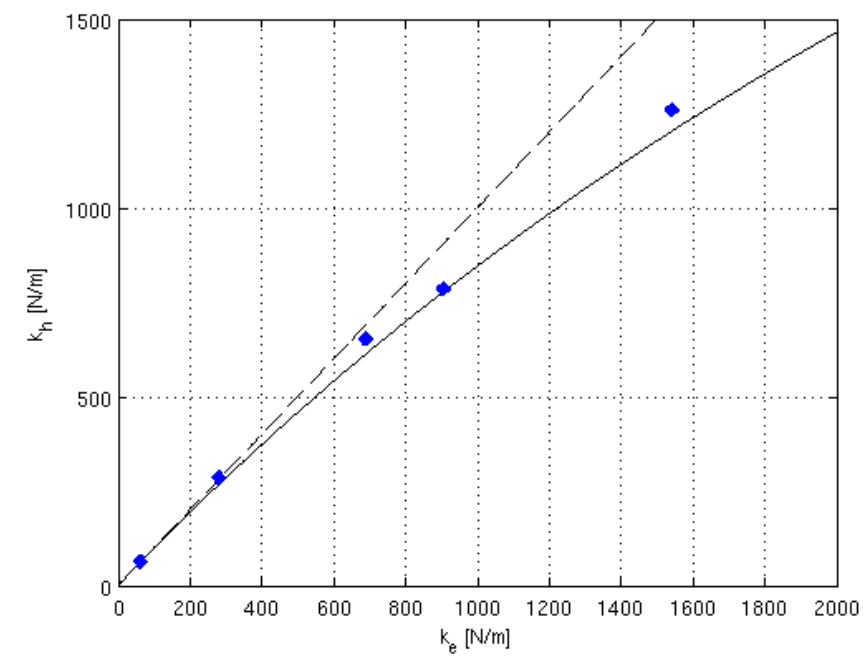

Fig. 9. Stiffness transmission of the teleoperation system from the environment (abscissa) to the human operator (ordinate)

For very stiff environments the transmitted stiffness converges to $k_{h, k_{e} \rightarrow \infty}=5475 \frac{N}{m}$, theoretically. In contact with a rigid surface of $\hat{k}_{e} \approx 50000 \frac{N}{m}$ and some unknown mass and damping, the transmitted stiffness was $\hat{k}_{h} \approx 5419 \frac{N}{m}$. With this environment the stability limit was reached. Note, that the torque control increases the robustness and dynamic range in contact but also reduces the ability to display large stiffnesses. However, a hard contact with an object is felt like a hard contact due to the high fidelity of the control architecture, that can track peaks in force and velocity (Fig. 7, left) that occur during impacts.

\section{SUMmARY AND CONCLUSION}

A high fidelity teleoperation control was presented integrating full state control of a flexible joint robot. An observation of master acceleration based on forces and torques is used to generate state references for the full state feedback controller of the slave. The direct force reflecting control architecture with full state reference is stable in contact with all passive environments tested, including rigid environments with a stiffness of more than $10000 \frac{\mathrm{N}}{\mathrm{m}}$. The passivity observer and the clutch was not needed for these environments and is rather a safety feature and theoretical justification for the non-passivity based, direct force reflecting control architecture. The teleoperation system lets the human operator feel structures on rigid objects, like the cavity of a dice, and transmits the stiffness of soft environments accurately.

The presented control approach, successfully combines and enhances methods from bilateral control and control of elastic joint robots. It takes advantage of rich sensory equipment in master and slave and knowledge of the dynamic models. It is also an encouraging control approach for comparable robots, like the DLR/KUKA Light-Weight Robot (LWR), in teleoperation applications without communication delay that demand high performance force feedback.

\section{REFERENCES}

[1] Alin Albu-Schäffer and Christian Ott. A unified passivity-based control framework for position, torque and impedance control of flexible joint robots. The International Journal of Robotics Research, vol. 26 no. 1:23-39, 2007.

[2] Goran A. V. Christiansson, Matthias Mulder, and Frans C. T. Van Der Helm. Slave device stiffness and teleoperator stability. In EuroHaptics 2006, pages 395-400, jul 2006.

[3] R. Daniel and P. McAree. Fundamental limits of performance for force reflecting teleoperation. In The International Journal of Robotics Research, 1998.

[4] U. Hagn, R. Konietschke, A. Tobergte, M. Nickl, S. Jörg, B. Kuebler, G. Passig, M. Gröger, F. Fröhlich, U. Seibold, L. Le-Tien, A. AlbuSchäffer, A. Nothelfer, F. Hacker, M. Grebenstein, and G. Hirzinger. DLR MiroSurge - a versatile system for research in endoscopic telesurgery. International Journal of Computer Assisted Radiology and Surgery, 2009.

[5] U. Hagn, T. Ortmaier, R. Konietschke, B. Kuebler, U. Seibold, A. Tobergte, M. Nickl, S. Jörg, and G. Hirzinger. Telemanipulator for remote minimally invasive surgery. IEEE Robotics \& Automation Magazine, 15(4):28-38, December 2008. DOI: 10.1109/MRA.2008.929925.

[6] B. Hannaford and R. Anderson. Experimental and simulation studies of hard contact in force reflecting teleoperation. In International Conference on Robotics and Automation, Phil., PA, 1988.

[7] Blake Hannaford and Jee-Hyan Ryu. Time domain passivity control of haptic interfaces. IEEE Transactions on Robotics and Automation, 18:1-10, 2002.

[8] R. Konietschke, G. Hirzinger, and Y. Yan. All singularities of the 9-DoF DLR medical robot setup for minimally invasive applications. In Advances in Robot Kinematics, 2006.

[9] D. Lawrence. Stability and transparency in bilateral teleoperation. In IEEE transactions on robotics and automation, 1993.

[10] L. Le-Tien, A. Albu-Schäffer, and G. Hirzinger. MIMO state feedback controller for a flexible joint robot with strong joint coupling. In Proceedings of the IEEE International Conference on Robotics and Automation (ICRA), pages 3824-30, Roma, Italy, April 2007. DOI: 10.1109/ROBOT.2007.364065.

[11] H. Mayer, I. Nagy, A. Knoll, E.U. Braun, R. Bauernschmitt, and R. Lange. Haptic feedback in a telepresence system for endoscopic heart surgery. Presence, 16(5):459-470, October 2007. DOI: 10.1162/pres.16.5.459.

[12] A. Okamura. Methods for haptic feedback in teleoperated robotassisted surgery. In Industrial Robot: An international Journal, 2004. 
[13] Ch. Ott, A. Albu-Schäffer, A. Kugi, S. Stramigioli, and G. Hirzinger. A passivity based Cartesian impedance controller - part I: Torque feedback and gravity compensation. In Proceedings of the IEEE International Conference on Robotics and Automation (ICRA), New Orleans, USA, 2004.

[14] Jee-Hwan Ryu, Jordi Artigas, and Carsten Preusche. A passive bilateral control scheme for a teleoperator with time-varying communication delay. Mechatronics, 20:812-823, 2010.

[15] Jee-Hwan Ryu, Carsten Preusche, Blake Hannaford, and Gerd Hirzinger. Time domain passivity control with reference energy following. IEEE Transactions on Control Systems Technology, 13 (5):737-742, 2005.

[16] A. J. Van der Schaft. L2-Gain and Passivity Techniques in Nonlinear Control. Springer-Verlag New York, Inc., Secaucus, NJ, USA, 1st edition, 1996.

[17] U. Seibold, B. Kuebler, and G. Hirzinger. Prototypic force feedbackinstrument for minimally invasive robotic surgery. In V. Bozovic, editor, Medical Robotics, pages 377-400. I-Tech Education and Publishing, Vienna, Austria, 2008.

[18] Pete Bradley. Shull and Günter Niemeyer. Force and position scaling limits for stability in force reflecting teleoperation. In ASME Dynamic Systems and Control Conference, 2008.

[19] Jean-Jacques Slotine and Weiping Li. Applied Nonlinear Control. Prentice Hall, October 1990.

[20] M. Tavakoli and R.D. Howe. The effect of joint elasticity on bilateral teleoperation. In International Conference on Intelligent Robots and Systems, San Diego, CA, USA, 2007.

[21] Sophie Thielmann, Ulrich Seibold, Robert Haslinger, Georg Passig, Thomas Bahls, Stefan Jörg, Mathias Nickl, Alexander Nothhelfer, Ulrich Hagn, and Gerd Hirzinger. Mica - a new generation of versatile instruments in robotic surgery. In Proceedings of IROS'10, the IEEE International Conference on Intelligent Robots and Systems, 2010.

[22] Andreas Tobergte, Patrick Helmer, Ulrich Hagn, Patrice Rouiller, Sophie Thielmann, Sébastien Grange, Alin Albu-Schäffer, François Conti, and Gerd Hirzinger. The sigma.7 haptic interface for mirosurge: A new bi-manual surgical console. In International Conference on Intelligent Robots and Systems (IROS), San Francisco, USA, 2011.

[23] Hagn U., Nickl M., Jörg S., Passig G., Bahls T., Nothhelfer A., Hacker F., Le-Tien L., Albu-Schäffer A., Konietschke R., Grebenstein M., Warpup R., Haslinger R., Frommberger M., and Hirzinger G. The DLR MIRO: A versatile lightweight robot for surgical applications. In Industrial Robot: An International Journal2008.

[24] B. Willaert, B. Corteville, D. Reynaerts, H. Van Brussel, and E.B. Vander Poorten. Bounded environment passivity of the classical positionforce teleoperation controller. In International Conference on Intelligent Robots and Systems (IROS), St. Louis, USA, 2009. 